\title{
Rare-event properties in a classical stochastic model describing the evolution of random unitary circuits
}

\author{
S. L. A. de Queirot* \\ Instituto de Física, Universidade Federal do Rio de Janeiro, \\ Caixa Postal 68528, 21941-972 Rio de Janeiro RJ, Brazil
}

(Dated: September 17, 2021)

\begin{abstract}
We investigate the statistics of selected rare events in a (1+1)-dimensional (classical) stochastic growth model which describes the evolution of (quantum) random unitary circuits. In such classical formulation, particles are created and/or annihilated at each step of the evolution process, according to rules which generally favor a growing cluster size. We apply a large-deviation approach based on biased Monte Carlo simulations, with suitable adaptations, to evaluate (a) the probability of ending up with a single particle at a specified final time $t_{f}$, and (b) the probability of having particles outside the light cone, defined by a "butterfly velocity" $v_{B}$, at $t_{f}$. Morphological features of singleparticle final configurations are discussed, in connection with whether the location of such particle is inside or outside the light cone; we find that joint occurrence of both events of types (a) and (b) drives significant changes to such features, signalling a second-order phase transition.
\end{abstract}

\section{INTRODUCTION}

In the study of quantum dynamics, one subject of interest is the propagation of quantum information. Random quantum circuits are useful in this connection, as they are minimally structured models which manage to capture universal properties pertaining to entanglement growth. The spreading of quantum operators under random circuit dynamics can be measured by the "out- oftime-order correlator" (OTOC) [1]. It has been established [2] that both in 1D and in higher spatial dimensions, operator spreading and the growth of the OTOC can be mapped to classical stochastic growth models. Here we make use of this correspondence, and study the time evolution of such classical systems, specializing to $1+1 \mathrm{D}$.

We are interested in rare events which occur with very small probabilities, also called large deviations 3 -5]. In order to achieve this we implement a large-deviation approach to Monte Carlo simulations which involves sampling according to a biased distribution [6, 7]. In this way, instead of focusing on typical events which, for standard simulations, occur with highest frequency, the peak of the associated probability distribution is shifted towards the atypical ones which are most relevant to our purposes. As described below, our basic implementation of such ideas closely follows recent work [8 11].

This paper is organized as follows. In Sec. Iwe present basic features of the model to be studied, as well as the calculational methods to be employed. Sec. III gives the results of our calculations. Section [V] is devoted to discussions and conclusions.

\section{MODEL AND METHODS}

Random unitary circuits (RUC) provide a simplified view of the quantum process of operator growth. Consider an operator $\mathcal{O}_{0}$ (e.g., a spin) initially localized near the origin; upon Heisenberg time evolution it will become $\mathcal{O}_{0}(t)=U^{\dagger}(t) \mathcal{O}_{0} U(t)$, acting on many sites. The "size" of $\mathcal{O}_{0}(t)$ is the size of the region in which $\mathcal{O}_{0}(t)$ does not commute with some local operator $Y_{x}$ at position $x$ [2]. Assuming that both $\mathcal{O}_{0}$ and $Y_{x}$ are Pauli-like operators, it can be shown that the operator spread is ruled by the correlator $\mathcal{C}(x, t)$ given by

$$
\mathcal{C}(x, t)=1-\operatorname{Tr} \rho_{\infty} \mathcal{O}_{0}(t) Y_{x} \mathcal{O}_{0}(t) Y_{x},
$$

where $\rho_{\infty}$ denotes the infinite-temperature Gibbs state; the second term, in which the operators are not timeordered, is the OTOC [2]. In such a case, the local operators can be taken to be literally spins localized on a lattice. The corresponding pictorial representation of the evolution process in one spatial dimension is shown in Fig 1. A spin resides on each blue lattice site. Each bond (depicted as a brown rectangle in the Figure) represents an independent Haar-random unitary, which acts on the joint Hilbert space of two adjacent spins of local Hilbert space dimension $q$ each. Here we shall only consider $q=2$ (Ising spins). Correlations propagate along the lattice via updating of bonds. In the quantum system each bond update contributes to $\mathcal{C}(x, t)$ of Eq. 1 .

The classical stochastic formulation in $1+1 \mathrm{D}$ which corresponds to (quantum) random unitary circuits (RUC) is as follows. Referring again to Fig. 1] we start with an infinite chain with sites denoted by integer numbers $\ell$ (the bond between sites $\ell$ and $\ell+1$ is referred to as "bond $\ell$ ", for short). Initially all sites are empty except for the "central" one where a single particle resides, so the occupation number $n_{\ell}(t=0)=\delta_{\ell, 0}$. This corresponds to the operator $\mathcal{O}_{0}$ of the quantum description.

The time evolution of this system proceeds via the update of bonds. This means changing the occupation numbers $\left\{n_{\ell}, n_{\ell+1}\right\}$ of the sites connected to bond $\ell$. 


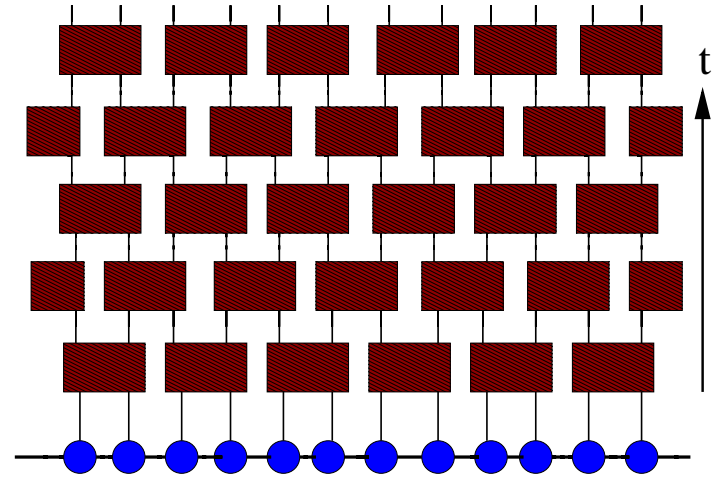

Figure 1. Lattices sites in blue. In the RUC problem spins reside on sites, and the brown rectangles represent independently Haar-random unitaries acting on the Hilbert space of two adjacent spins. In the classical growth model the rectangles denote bonds between adjacent lattice sites; the latter's occupation numbers evolve in time. At each $t$ the corresponding bonds are updated. The staggered placement of rectangles corresponds, in the classical model, to the rule that alternating halves of the lattice are sequentially updated according to Eq. (2) (adapted from Fig. 1 of Ref. 2).

An elementary step consists of a simultaneous update of, say, all even bonds $2 \ell$; the next step is then a simultaneous update of all odd bonds $2 \ell-1$. So all sites are examined once, and their occupations possibly changed, at each step. See Fig. 1.

The update rules are as follows:

(R1) $\left\{n_{\ell}, n_{\ell+1}\right\}(t+1)=\{0,0\}$ if $\left\{n_{\ell}, n_{\ell+1}\right\}(t)=\{0,0\}$.

(R2) if $\left\{n_{\ell}, n_{\ell+1}\right\}(t)$ is any of $\{1,0\},\{0,1\}$, or $\{1,1\}$ then

$$
\left\{n_{\ell}, n_{\ell+1}\right\}(t+1)=\left\{\begin{array}{l}
\{1,0\} \text { with probability } p \\
\{0,1\} \text { with probability } p \\
\{1,1\} \text { with probability } 1-2 p
\end{array}\right.
$$

where $p=1 /\left(q^{2}+1\right)[2]$. Here, for Ising spins, $p=1 / 5$. In this case, one may pictorially view the occupation numbers of the classical formulation as akin to the individual spin states of its quantum counterpart. Although particle annihilation is possible, the above rules favor a cluster growing in size with time.

The ensemble-averaged, time-dependent, length of the cluster, which corresponds to the "size" of the OTOC, grows linearly in time with a "butterfly" speed $v_{B}$. In $1+1 \mathrm{D}$ the exact results of Ref. 2 show that

$$
v_{B}=\frac{q^{2}-1}{q^{2}+1},
$$

so $v_{B}=3 / 5$ in the Ising case. $v_{B}$ gives the opening of the light cone associated with the average operator spread.

Here we investigate the statistics of two species of atypical events occurring in the evolution process described above.

(a) The probability of ending up with a single particle at a "final" time $t_{f}$. (b) The probability and morphology of "superluminar" configurations, that is, ones which extend beyond the light cone boundaries defined by $x= \pm v_{B} t$.

The physical motivation for the study of the above quantities can be understood as follows. Firstly, given the update rules spelt in Eq. (2), and with $p=1 / 5$, the minimal final value $N_{f} \equiv N\left(t_{f}\right)=1$ will only be achieved through some very particular set of concurring, entropy-reducing, fluctuations occurring along the evolution process. Thus one would expect the intermediate shapes assumed by the cluster at $t<t_{f}$ to reflect the cumulative contributions of such fluctuations. Conversely, realizations with larger $N_{f}$ would presumably be free of effects of the sort. Secondly, given that $v_{B}$ signals the speed of growth of the ensemble-averaged size of the cluster, excursions outside the light cone should result from the accumulation of rare cooperative effects of independent fluctuations. Again, the question arises of how the accrual of such effects leaves an imprint on the evolving cluster shapes.

Through a quantitative investigation, here we attempt to draw connections between rare events of types (a) and (b), and the morphological characteristics exhibited in the evolution of the clusters associated with such events. In order to do so, we first consider the statistics of $N_{f}=1$ events in Sec. IIIB, general features of super-luminar configurations are given in Secs IIIC 1 for $N_{f}=1$, and Sec. IIIC2 for $N_{f}>1$. We are then in a position, in Sec. IIIC3, to look at the shape and size of clusters with $N_{f}=1$, and check how those properties depend on whether the location of the single remaining particle is supra- or sub-luminar.

A configuration with $N_{f}=1$ would correspond, in the quantum RUC model, to the Heisenberg evolution of an operator in such a way that it ends up having the same "size" with which it started (i.e., the length over which it spreads at the end is basically the same as the initial one, albeit that it may be shifted from the origin). Similarly, a super-luminar configuration would correspond to a quantum evolution process in which the operator correlations spread over atypically long distances. Since both types of events in the quantum domain would exhibit such very specific features, one can expect them to be associated with other distinctive traits belonging exclusively to the quantum formulation.

We briefly describe our calculational methods as applied to case (a). With $P_{N}(t)$ being the probability of having a configuration with $N$ particles in total after $t$ lattice updates, the rules given above allow for $1 \leq N(t) \leq 2 t$ with the most probable value $N_{m p}(t) \approx t$. While the region close to the peak of the distribution is well-described, the low-probability tails are much less richly sampled, which tends to compromise the accuracy of evaluation of $P_{1}\left(t_{f}\right)$ if $t_{f} \gg 1$.

Following Refs. 8-11, we consider a sequence $Y=$ $\left(y^{(0)}, y^{(1)}, \ldots y^{\left(t_{f}\right)}\right)$ of configurations (each consisting of a collection of occupation numbers $\left.\left\{n_{\ell}(t)\right\}\right)$, where the transitions $y^{(t)} \rightarrow y^{(t+1)}$ follow the update rules; one 
always starts with $\left\{n_{\ell}(0)\right\}=\delta_{\ell 0} . \quad Y$ is called a history. The quantity of interest for a given history $Y$ is $N_{f}(Y) \equiv N\left(t_{f}\right)$, obtained from the associated $y^{\left(t_{f}\right)}$. With standard sampling, each history occurs with its natural probability $R(Y)$.

In the large deviation Monte-Carlo method, sample sequences are generated according to a biased distribution $R_{\theta}(Y)$, in which the probability is

$$
R_{\theta}(Y)=\frac{1}{Z(\theta)} R(Y) e^{-N_{f}(Y) / \theta}
$$

where $\theta$ is a fictitious temperature and $Z(\theta)$ is a normalization factor. For $\theta>0$ histories with smaller values of $N_{f}(Y)$ will be favored; the peak of the biased distribution is thus shifted towards such configurations with low final density.

One can transform between the biased distribution $P_{\theta}\left(N_{f}\right)$ and the unbiased one $P\left(N_{f}\right)$ which is of ultimate interest by noting that [1]

$$
\begin{gathered}
P_{\theta}\left(N_{f}\right)=\sum_{Y} R_{\theta}(Y) \delta_{N_{f}(Y), N_{f}}= \\
=\frac{e^{-N_{f} / \theta}}{Z(\theta)} \sum_{Y} R(Y) \delta_{N_{f}(Y), N_{f}}=\frac{e^{-N_{f} / \theta}}{Z(\theta)} P\left(N_{f}\right) .
\end{gathered}
$$

One generates the biased distributions $P_{\theta}\left(N_{F}\right)$ according to a standard Metropolis-Hastings algorithm, through the analysis of a Markov chain of histories $\left\{Y\left(t_{\mathrm{MC}}\right)\right\}$, where the Monte Carlo times $t_{\mathrm{MC}}=0,1,2 \ldots$ denote successive updates $Y\left(t_{\mathrm{MC}}\right) \rightarrow Y\left(t_{\mathrm{MC}}+1\right)$. For the update, one generates a trial history $Y_{\text {trial }}$, which is accepted as the next element $Y\left(t_{\mathrm{MC}}+1\right)$ of the Markov chain with the Boltzmann-like probability

$$
A\left(Y \rightarrow Y_{\text {trial }}\right)=\min \left(1, e^{-\left\{N_{f}\left(Y_{\text {trial }}\right)-N_{f}\left[Y\left(t_{\mathrm{MC}}\right)\right]\right\} / \theta}\right)
$$

If $Y_{\text {trial }}$ is not accepted, then $Y\left(t_{\mathrm{MC}}+1\right)=Y\left(t_{\mathrm{MC}}\right)$. Such transition rules indeed give probabilities as in Eq. (4) and, as is well known, represent the BoltzmannGibbs equilibrium distribution for a system at temperature $\theta[8]$.

The lattice update rules of Eq. (2) make use of (pseudo)-random numbers (RN), to decide which among the possible outcomes of the examination of each bond is chosen. In the large deviation approach, the systematics of generating trial configurations is as follows [8 11].

(i) Instead of generating an $\mathrm{RN}$ each time one is needed, a collection of RNs is computed before running the actual simulation, i.e., prior to generating a history $Y$, and stored in a "list", or vector, $\xi$ 11]. This list is then consulted in ascending order, say, each time an RN is required in the build-up of $Y$.

(ii) For a given $t_{\mathrm{MC}}$ with its associated $\xi\left(t_{\mathrm{MC}}\right)$ and $Y\left(t_{\mathrm{MC}}\right)$, a trial vector $\xi_{\text {trial }}$ is generated by randomly replacing a fraction $f$ of the components of $\xi\left(t_{\mathrm{MC}}\right)$ with freshly-evaluated RNs; this is used in simulating a trial history $Y_{\text {trial }}$. (iii) If the latter is accepted according to Eq. (6), then one makes $\xi\left(t_{\mathrm{MC}}+1\right)=\xi_{\text {trial }}$. Otherwise a new $\xi_{\text {trial }}$ is generated, starting again from $\xi\left(t_{\mathrm{MC}}\right)$, with the respective $Y_{\text {trial }}$ being tested via Eq. (6).

As a rule of thumb, it is customary to choose $f$ in step (ii) above in such a way that approximately half the trial histories are accepted [11].

In this way one probabilistically guides the path followed by the successive $Y\left(t_{\mathrm{MC}}\right)$, towards a region of configuration space where realizations of the evolution with the desired features are more frequent (relative to the unbiased case, which corresponds to $\theta \rightarrow \infty)$.

By choosing a number of suitable values of $\theta$ one can generate a set of biased distributions, each of which is smooth for a specific range of the variable of interest, in such a way that pairs of said ranges overlap each other to some (not necessarily very broad) extent. One then uses Eq. (5), and its straightforward generalization for pairs of finite $\theta$ values, to fit the respective $Z(\theta)$ by requiring each pair of adjacent distributions to match one another within the corresponding overlap range [8, 10, 11]. Starting from the unbiased distribution restricted to the range where it is most accurate, one then fully reconstructs its full extent by joining smoothly-shaped pieces.

We illustrate the methods just described by checking that the distributions studied here behave in line with the large deviation principle [3 5]. We do this by evaluating the size-dependent rate function 11,12 . It can be seen that the size-like parameter in our problem is the final time $t_{f}$ [2]. It follows from Eq. (2) that the maximum number of particles at time $t_{f}$ equals $2 t_{f}$. So we take the density-like quantity $N\left(t_{f}\right) / 2 t_{f}$ as the scaled variable to be used when comparing the $P\left(N\left(t_{f}\right)\right)$ for different final times $t_{f}$. Accordingly, we define the rate function $\Phi\left(N\left(t_{f}\right)\right)$ as:

$$
\Phi\left(N\left(t_{f}\right)\right)=-\frac{1}{2 t_{f}} \ln P\left(N\left(t_{f}\right)\right) .
$$

According to the large-deviation principle the size dependence of the rate function should vanish as the system "size" increases [11, 12].

Figure 2 shows that our numerical data do behave in the expected way, with curves for various $t_{f}$ becoming closer to one another as $t_{f}$ increases. For evaluation of the $P\left(N\left(t_{f}\right)\right)$ we used biased distributions with both positive and negative fictitious temperatures $\theta$, corresponding respectively to the left and right tails of the full curves shown. We generally took $\theta= \pm 1$ and 2 , plus $\theta=0.8$ for $t_{f}=120$ and 1.5 for $t_{f}=80$, to complement data for the left tail which is of particular interest for single-particle final configurations (see Sec. IIIB below).

Further comments on details of the implementation of the approach outlined above, especially those regarding specific aspects of the processes studied here, are given in Sec. III 


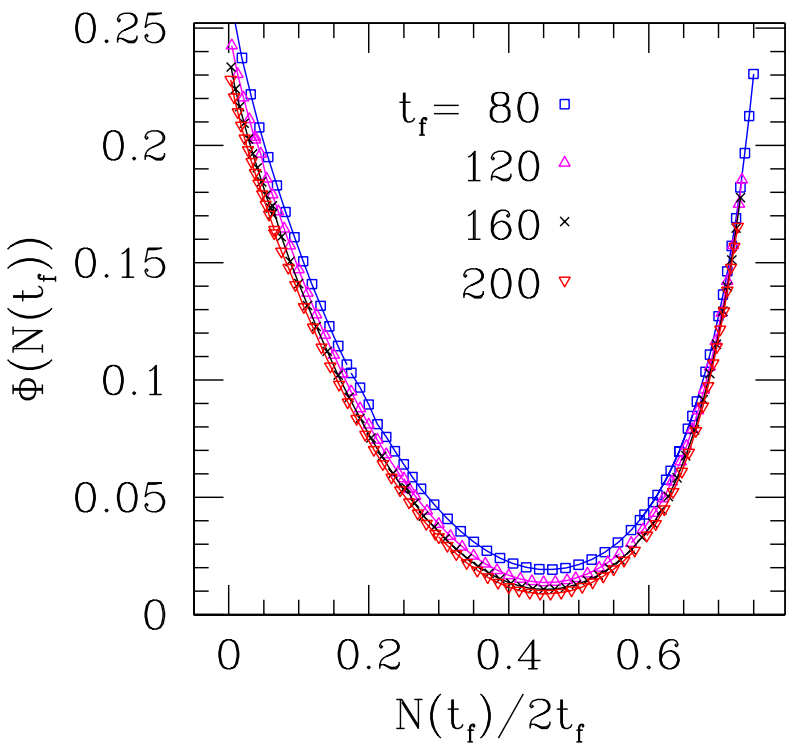

Figure 2. The rate function $\Phi\left(N\left(t_{f}\right)\right)$ defined in Eq. (7) for several values of $t_{f}$. The distributions $P\left(N\left(t_{f}\right)\right)$ were evaluated with $10^{8}$ samples each. In all cases biased distributions, generally with $\theta= \pm 1$ and 2 , were used additionally to the unbiased ones (see text).

\section{RESULTS}

\section{A. Introduction}

Examination of the rules given in Eq. (2) shows that, for a given history, the number and places of occurrence of non-trivial bond updates [i.e. differing from $\{0,0\} \rightarrow\{0,0\}]$ are not a priori fixed. At time $t$ the location $\ell$ of each such update (requiring an RN to decide its outcome) depends on the results of previous updates. This is in contrast with the cases analysed in Refs. 8 11, where the total number of RNs required for generating each configuration $y^{(t)}$ of a history is fixed. In such cases one can view each elementary $\mathrm{RN}$ replacement, when building $\xi_{\text {trial }}$ from $\xi\left(t_{\mathrm{MC}}\right)$, as analogous to changing the probability of a given (local) spin flip when simulating a magnetic system 11]. The complementary fact that a non-replaced $\mathrm{RN}$ then corresponds to a certain spin which undergoes the same process, at the same "microscopic" time $t$, as in the previous history, is in line with the idea that replacing a few RNs amounts to a "small" local change to the process being simulated.

In the present case, the replacement of a single $\mathrm{RN}$ may have effects throughout the extension of the light cone associated with the bond where it occurs. Thus the "local spin-flip" analogy invoked in previous cases is not obviously applicable here. Even so, we have found that following rules (i)-(iii) given at the end of Sec. II can be enough to yield the desired outcome of producing biased sampling as defined in Eq. (4). Furthermore, we have seen that replacing a fraction $f$ between $1.25 \%$ and $2.5 \%$ of the components of a vector $\xi$ in simulations of the RUC model has a similar effect, in terms of the acceptance rate of trial configurations via Eq. (6), to replacing of order $10 \%$ of the corresponding vector for other cases [8]11. This is very likely related to the nonlocal effects of replacing RNs in the current model, alluded to above.

In practice we generated an initial $\xi$ slightly overdimensioned, with $M=3 t_{f}^{2} / 4$ components (corresponding to a matrix with $t_{f} / 2$ lines, $3 t_{f} / 2$ columns in $\left.1+1 \mathrm{D}\right)$, so one could be sure that the end of the list would not be reached during the build-up of a history $Y$. Each time a history is accepted, we record the locations $\{\ell, t\}$ where RNs were used. For the following trial history we randomly replace a fraction $f$ of the RNs associated with the set of previously used locations. In doing so we are attempting to realize the concept of a "local" change in the closest possible way, given the update rules.

In our simulations of RUC evolution the process always starts with the same configuration, namely $n_{\ell}(t=0)=$ $\delta_{\ell, 0}$. With standard (unbiased) sampling, this means that there is no need to wait for "thermalization". This is in contrast with other problems described via Monte Carlo methods, where in general one starts with arbitrary initial conditions, and must run the simulation until such a "microscopic" time $t_{R}$ when those conditions are "forgotten", and the system relaxes into a steady state.

However, for biased sampling as described above, one still has a different sort of equilibration, related to the fictitious temperature $\theta$, as the steps of the Markov chain are correlated via Eq. (6). As stated in Sec. II rules (i)-(iii) given there ensure that the path followed by the successive $Y\left(t_{\mathrm{MC}}\right)$ leads the system to the appropriate region of configuration space. So it takes a finite amount of Monte Carlo time $t_{\mathrm{MC}}$ for such relaxation process to take place. We illustrate this in Fig. 3, where for each curve we ran $n_{s}=10^{4}$ independent Markov chains up to $t_{\mathrm{MC}}=4000$, each chain composed of histories starting with a single particle at the origin and evolving to $t_{f}=$ 40. At successive intervals $\Delta t_{\mathrm{MC}}=100$ we evaluated the average $\left\langle N_{f}\left(\theta, t_{\mathrm{MC}}\right)\right\rangle$ over the $n_{s}$ samples.

As anticipated, the curve corresponding to unbiased sampling shows no relaxation effects.

Although our main interest here is the limit of low $N_{f}$, corresponding to $\theta>0$, for completeness we looked at thermalization in cases where the bias is toward high final densities, enabled via making $\theta<0$. The curves for $\theta=$ -1.0 and -3.5 in Fig. 3 illustrate that the approach to stationarity is qualitatively similar to that for $\theta>0$, only it is from below. In summary, the bias always takes the system's average density away from the unbiased value given by the blue points in the Figure.

The finite- $\theta$ curves fit reasonably well to

$$
\left\langle N_{f}\left(\theta, t_{\mathrm{MC}}\right)\right\rangle=N_{f}^{0}(\theta)+a_{\theta} e^{-t_{\mathrm{MC}} / \tau_{\mathrm{MC}}(\theta)} .
$$

The adjusted decay times are $\tau_{\mathrm{MC}}(\theta)=540(35)$, $204(11), 93(10)$ for $\theta=1.0,2.0$, and 3.5 respectively. 


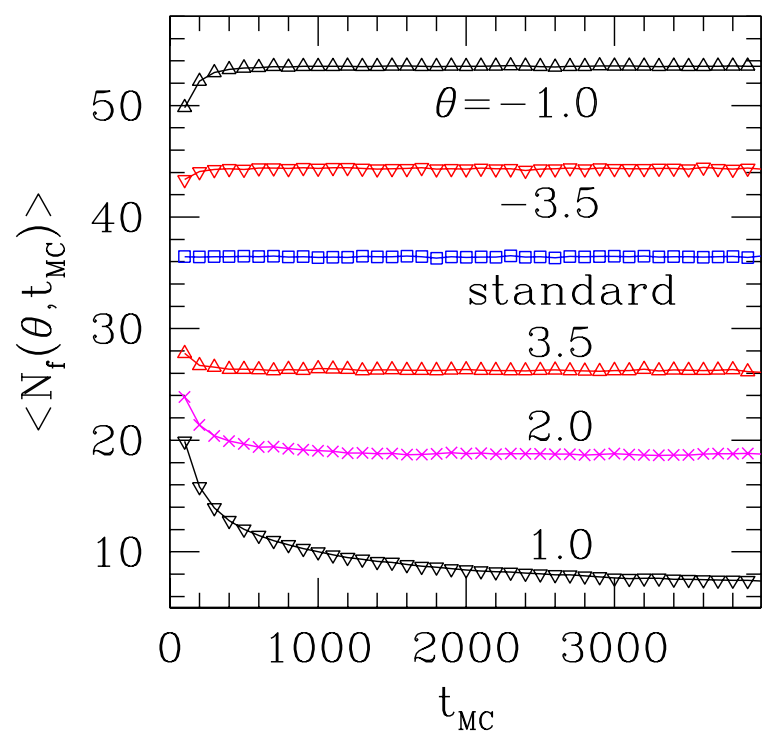

Figure 3. $\left\langle N_{f}\left(\theta, t_{\mathrm{MC}}\right)\right\rangle$ are averages, at fixed $t_{\mathrm{MC}}$, over $10^{4}$ independent histories of RUC evolving up to $t_{f}=40$, for various fictitious temperatures $\theta$. The "standard" label corresponds to unbiased sampling, $\theta \rightarrow \infty$. For finite $\theta$ trial vectors $\xi_{\text {trial }}$ have a fraction $f=0.025$ of the components of previous $\xi_{\mathrm{MC}}$ randomly replaced (see text).

For $\theta=-1.0$ and -3.5 one has $\tau_{\mathrm{MC}}(\theta)=104(2)$ and $81(10)$. For $\theta=1.0$ a much improved fit is found by assuming a superposition of long- and short-lived equilibration processes,

$$
\begin{gathered}
\left\langle N_{f}\left(\theta, t_{\mathrm{MC}}\right)\right\rangle=N_{f}^{0}(\theta)+a_{\theta} e^{-t_{\mathrm{MC}} / \tau_{\mathrm{MC}}^{1}(\theta)}+ \\
+b_{\theta} e^{-t_{\mathrm{MC}} / \tau_{\mathrm{MC}}^{2}(\theta)} \quad[\theta=1.0]
\end{gathered}
$$

where $a_{\theta}$ and $b_{\theta}$ turn out to be of the same order of magnitude, $\tau_{\mathrm{MC}}^{1}(\theta)=1076(27), \tau_{\mathrm{MC}}^{2}(\theta)=128(4)$. We applied Eq. (9) to cases with longer final ("real") times $t_{f} \leq 200$ as well, which will turn out to be relevant in what follows, and found that for a range of $\theta \geq 1$ the longest Monte-Carlo decay time $\tau_{\mathrm{MC}}^{1}(\theta)$ always remains in the range $(1-2) \times 10^{3}$. So in our simulations we generally decided to discard the first 8,000 MC steps in order to avoid inclusion of non-equilibrated samples. Since one needs at the very least some $10^{6}$ truly equilibrated samples to generate suitably smooth probability distributions for application of the large-deviation MC approach, it is seen that avoiding relaxation effects takes less than $1 \%$ of the total computational effort involved.

\section{B. Statistics of $N_{f}=1$ configurations}

In Fig. 4 we show the probability distribution $P_{N}\left(t_{f}\right)$ for $t_{f}=40$, evaluated both with standard (unbiased) sampling and with $\theta=2.0$. For the latter case, taking

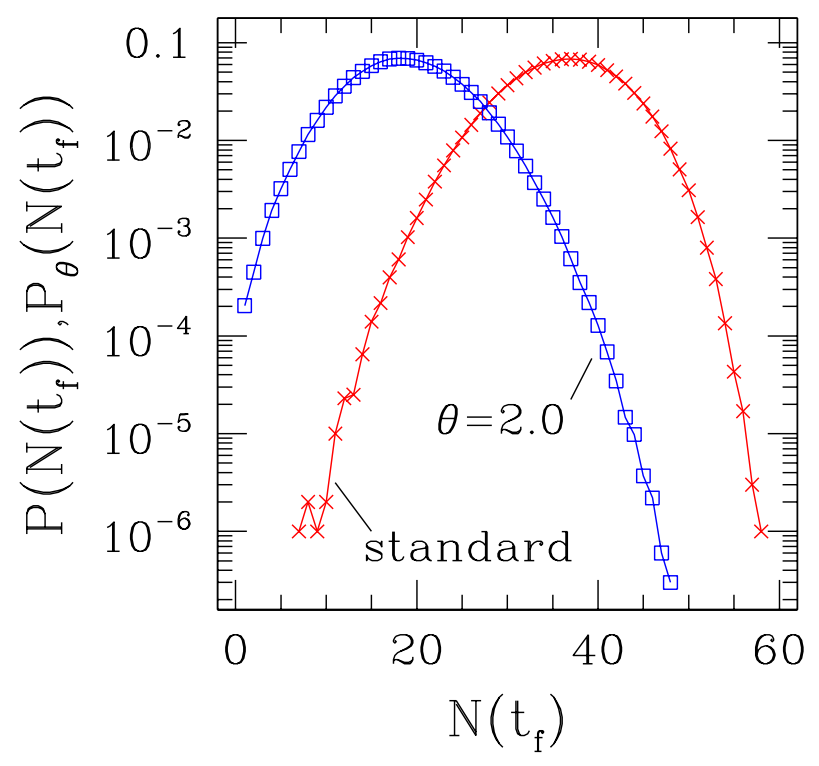

Figure 4. Probability distributions $P\left(N\left(t_{f}\right)\right), P_{\theta}\left(N\left(t_{f}\right)\right)$, for total number of particles after $t_{f}=40$ lattice updates. The "standard" label denotes unbiased sampling, $\theta \rightarrow \infty$. Effective length of Markov chain corresponds to $10^{6}$ independent samples for unbiased case, $10^{7}$ update attempts (see Sec. II) for $\theta=2.0$.

into account the results exhibited in Fig. 3 we discarded start-up simulation data corresponding to $t_{\mathrm{MC}} \leq 2000$.

It is seen that the standard distribution loses accuracy for $N \lesssim 20$. As already mentioned in Sec. III in line with the usual procedures of the large-deviation approach [811] we search for a range of $N\left(t_{f}\right)$ of common support for the biased and unbiased distributions, where both exhibit smooth features. Referring to Fig. 4, we find that this corresponds to $20 \lesssim N\left(t_{f}\right) \lesssim 35$. Within this range we match both distributions via Eq. (5), thus extracting an accurate estimate of $Z(\theta)$.

Fig. 5 shows the result of the matching procedure using $25 \leq N\left(t_{f}\right) \leq 32$, from which one gets $Z^{-1}(\theta)=$ $958(5) \times 10^{3}$. This in turn gives $P_{1}\left(t_{f}\right)=3.51(2) \times 10^{-10}$. The largest source of systematic uncertainty for the latter quantity seems to be the matching process itself, rather than the underlying accuracy of [the smooth regions of] the distributions, which is quite satisfactory when one uses $10^{6}-10^{7}$ samples as here.

From a Gaussian fit to the $[20-50]$ data range for the standard simulation data of Fig. 4, one finds the extrapolated estimate $P_{1}^{G}\left(t_{f}\right)=5.9 \times 10^{-10}$. This is in line with the idea that near the lower bound the distribution must fall more steeply than predicted by a Gaussian picture, which assumes the curve to extend indefinitely to lower values. However, the discrepancy is only by a factor of order two, suggesting that the Gaussian description works reasonably well as a first approximation.

For longer final times $t_{f}$, one must resort to values 


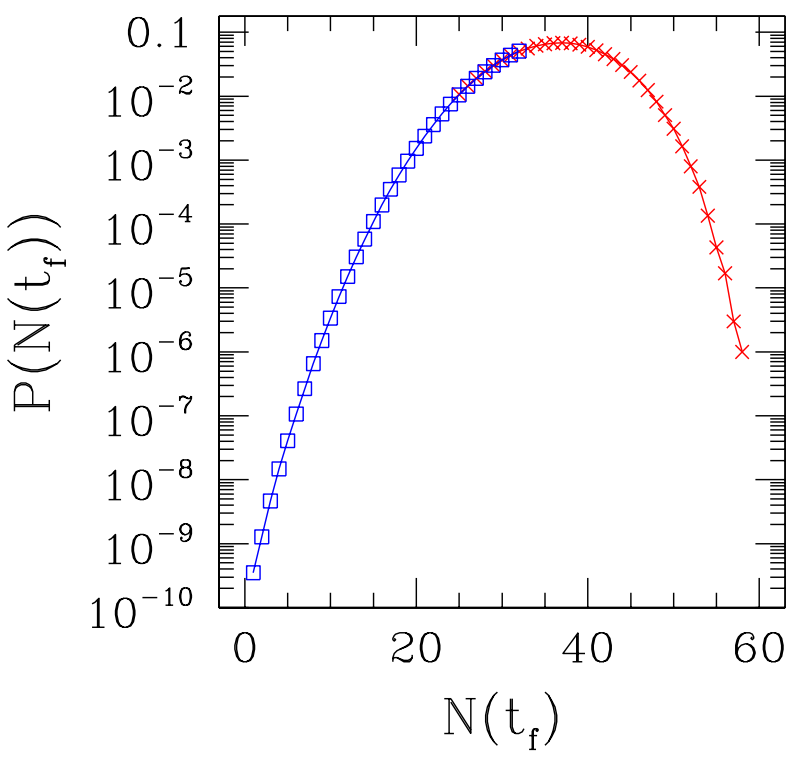

Figure 5. Result of matching probability distributions $P\left(N\left(t_{f}\right)\right)$ and $P_{\theta}\left(N\left(t_{f}\right)\right)$ displayed in Fig. 4 following Eq. (5). Symbol labels are the same as that Figure. Data are superimposed for $25 \leq N\left(t_{f}\right) \leq 32$, corresponding to the range actually used for the matching of curves.

of $\theta<2$ in order to have direct access to $P_{1}\left(t_{f}\right)$. The combination of ballistic drift and diffusive broadening of the front [2] suggests a semiquantitative estimate, based again on a Gaussian approximation, giving what is expected to be an upper bound for $P_{1}\left(t_{f}\right)$ :

$$
P_{1}^{G}\left(t_{f}\right) \approx a t_{f}^{-1 / 2} \exp \left(-b t_{f}\right) .
$$

Using $t_{f}=40$ data from Fig. 4 plus results from an unbiased simulation for $t_{f}=200$ in Eq. (10), one gets $a \approx 0.0682, b \approx 0.418$. Thus, for example, $P_{1}^{G}(200)=$ $2.37 \times 10^{-39}$.

We have numerically estimated $P_{1}\left(t_{f}\right)$ for a few $t_{f} \leq$ 200 , which required use of $\theta$ values as low as 0.8 . The results are exhibited in Fig. 6. together with the form given in Eq. (10). It can be seen that the latter indeed acts as a rather stringent upper bound for the former, exceeding the numerical result by no more than a single order of magnitude for $t_{f}=200$.

\section{Super-luminar configurations}

We wish to investigate configurations in which one or more particles reach a region of space outside the characteristic light cone. Thus, for evolution up to a final time $t_{f}$ we search for occupied sites with coordinates $x$ such that

$$
|x|>v_{B} t_{f},
$$

where $v_{B}=0.6$ is the "butterfly" speed in this case [2].

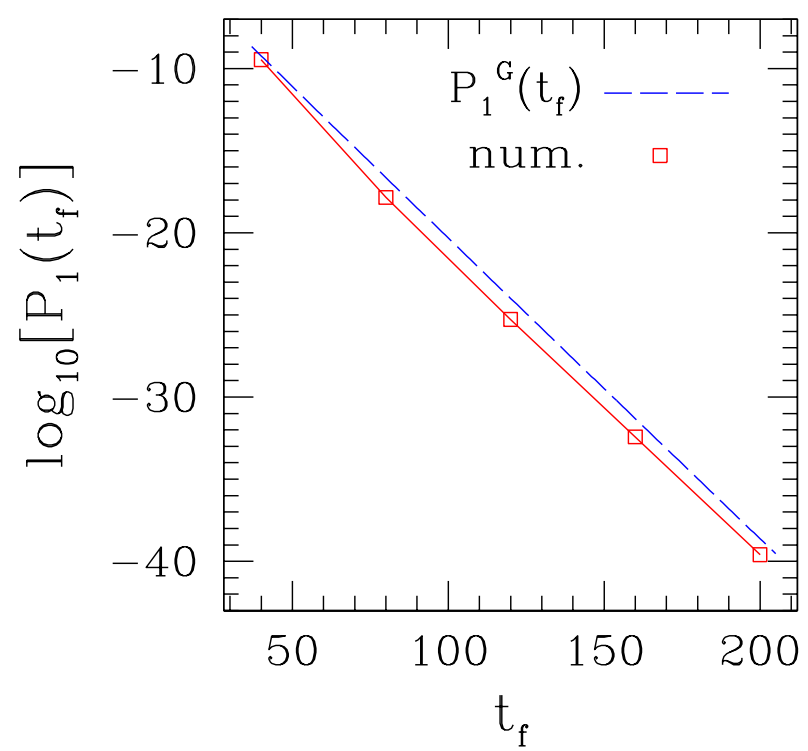

Figure 6. Red squares are numerical results for probability $P_{1}\left(t_{f}\right)$ of finding a single remaining particle at $t=t_{f}$, for $t_{f}=40,80,120,160$, and 200. Uncertainties are smaller than symbol sizes. The dashed blue line is the Gaussian approximation, Eq. (10).

\section{1. single-particle configurations}

Initially, for ease of visualization we concentrated on the subset of configurations with $N_{f}=1$. In what follows we take $t_{f}=40$. We saw in Sec. IIIB that $P_{1}\left(t_{f}\right)=3.51(2) \times 10^{-10}$. In order to generate richer statistics for the low- $N_{f}$ end of the distribution, we used a biased simulation with $\theta=1.5$ and $N_{s}=10^{9} \mathrm{MC}$ steps in all, and selected the resulting configurations which ended up with $N_{f}=1$. On account of the bias we obtained $P_{\theta=1.5}\left(N_{f}=1\right)=1.9 \times 10^{-3}$, which translates to the size of the restricted ensemble being $\approx 1.9 \times 10^{6}$ configurations. Note that, since each of the chosen configurations has its intrinsic probability biased by the same factor, see Eq. (4), they all have the same weight in the restricted ensemble.

Fig. 7 shows the site-dependent (conditional) probability $P_{1}^{c}\left(x_{f}, t_{f}\right)$ of having the single remaining particle at position $x_{f}$. Given the size of the restricted ensemble, we cannot access values of $P_{1}^{c}\left(x_{f}, t_{f}\right) \lesssim 10^{-6}$. Consequently the super-luminar region $|x|>24$ is out of direct reach. The data for pairs of neighboring sites show a tendency towards degeneracy, to which we will return later on in Sec. III 2 For the moment we note that, after averaging for such short-range effects, the available data are remarkably well fitted by a Gaussian function of width $\sigma=4.62(2)$, shown as a blue line in the Figure. Its peak lies within less than half a lattice spacing of the origin. Similarly to the case discussed in connection with Eq. (10), we assume that the Gaussian description re- 


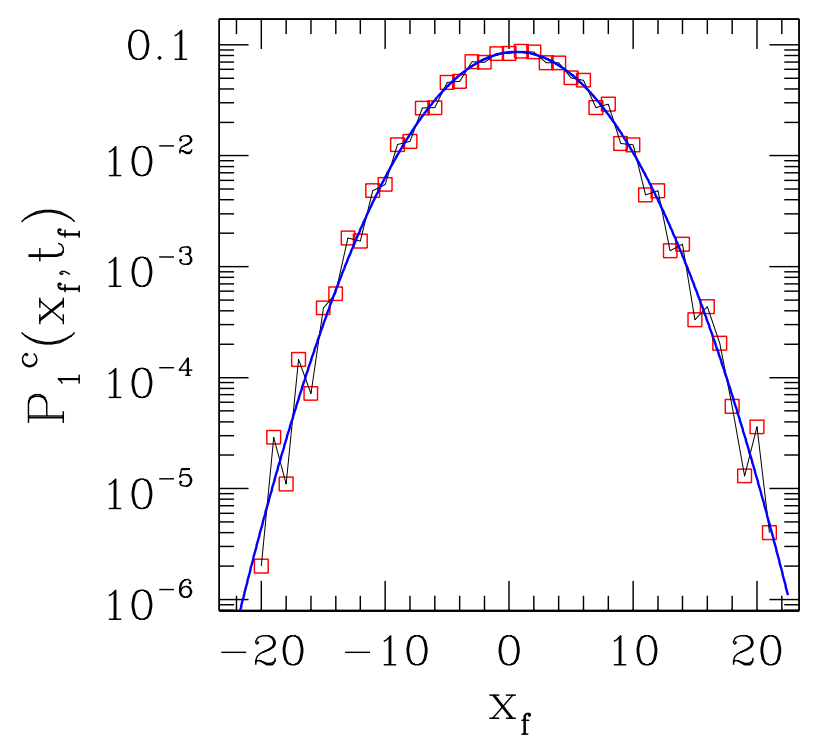

Figure 7. Conditional probability $P_{1}^{c}\left(x_{f}, t_{f}\right)$ of having a particle at position $x_{f}$, given that only a single particle remains, against position $x_{f}\left(\right.$ at $\left.t_{f}=40\right)$. Restricted ensemble has $\approx 1.9 \times 10^{6}$ independent configurations. Blue line is a Gaussian fit to the data (see text).

mains a reasonable one away from the region for which it was originally fitted. Accepting this, one finds that the region contained beyond the boundaries of the light cone, i.e. $P_{1}^{c}\left(x_{f}>v_{B} t_{f}, t_{f}\right)$ corresponds to the area below the standard error function beyond $|x| \approx 5 \sigma$, which is $\approx 10^{-12}$. At this point one can return to the original (unbiased) distribution and estimate, from the data exhibited in Fig. 5. $P_{1}\left(\left|x_{f}\right|>v_{B} t_{f}\right) \approx 3.5 \times 10^{-22}$.

\section{Configurations with $N_{f} \geq 1$}

We now consider the ensemble of all possible final configurations, irrespective of the total number of particles remaining. We took $t_{f}=40$ and measured the site occupation averages $\langle\rho(x)\rangle$ (ensemble-averaged local site occupations, with unbiased sampling) at $t_{f}$ over $10^{7}$ independent samples, with results shown in Fig. 8. Note a degeneracy of ensemble-averaged densities on pairs of neighboring sites. This was already pointed out in connection with Fig. 7 and is a consequence of the symmetries embedded in the evolution rules given through Eq. (2). Such degeneracy is broken (to a quantitatively small degree) by the introduction of a directional bias, as shown below.

With $N_{\text {out }} \equiv N\left(|x|>v_{B} t_{f}\right)$ being the integrated density on sites outside the light cone, and $N_{\text {all }}$ the corresponding quantity over the whole lattice, the relevant fraction is $f_{\text {out }} \equiv N_{\text {out }} / N_{\text {all }}$.

The data exhibited in Fig. 8 give $f_{\text {out }}=0.07218 \ldots$

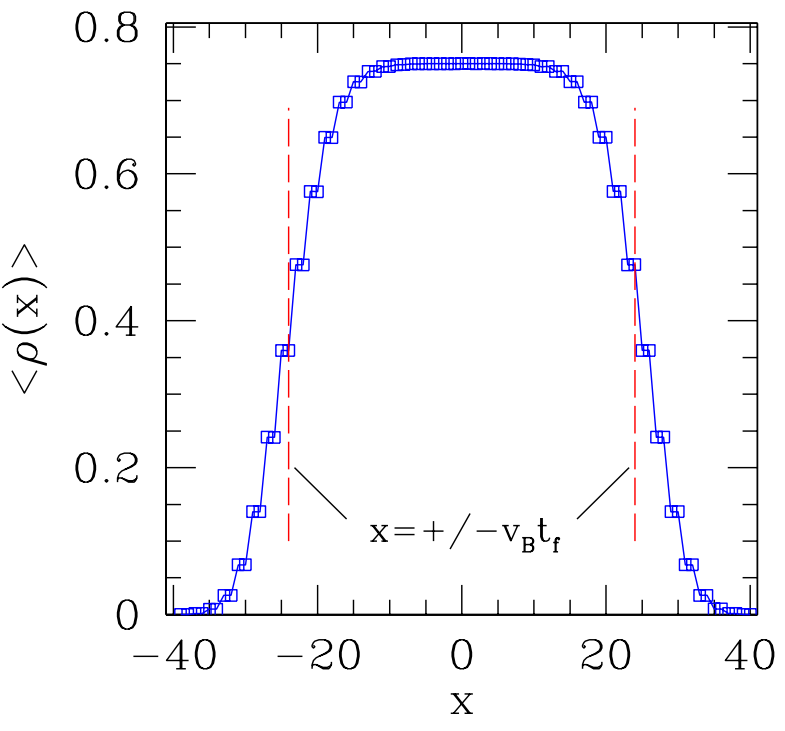

Figure 8. Ensemble-averaged local site occupations $\langle\rho(x)\rangle$ at $t_{f}=40$ against position $x$, for $10^{7}$ independent samples. Dashed red lines mark boundaries of light cone at $x= \pm v_{B} t_{f}= \pm 24$.

Increasing $t_{f}$ makes the rate of decrease of $\langle\rho(x)\rangle$ around $x=v_{B} t_{f}$ become steeper. The midway points where the density becomes half of the plateau value $\langle\rho(0)\rangle$ coincide with $|x|=v_{B} t_{f}$ to a very good extent, as is already the case depicted in Fig. 8 . For $t_{f}=80$ one gets $f_{\text {out }}=$ $0.05373 \ldots$...

We wish to generate statistical samples containing many configurations with occupied sites outside the light cone. Defining $N_{\text {out }}^{L}=N\left(x<-v_{B} t_{f}\right), N_{\text {out }}^{R}=N(x>$ $\left.v_{B} t_{f}\right)$, with the corresponding occupation fractions $f_{\text {out }}^{L}$, $f_{\text {out }}^{R}$, one possible way to enhance one of the $f_{\text {out }}^{L, R}$ is to introduce a directional bias in the sampling process. In analogy with the fictitious temperature $\theta$ introduced in Eq. (4), which couples to the number of particles $N\left(t_{f}\right)$, we introduce a second "temperature" $\tau$ coupled to the position of the leftmost occupied site at $t_{f}, x_{\mathrm{lm}}^{f}$.

$$
R_{\tau}(Y)=\frac{1}{Z_{\operatorname{lm}}(\tau)} R(Y) e^{-x_{\operatorname{lm}}^{f}(Y) / \tau}
$$

where $Z_{\operatorname{lm}}(\tau)$ is once more a suitable normalization constant. This is the simplest way to incorporate the desired sort of bias. Of course different choices could be made for the quantity to be coupled to $\tau$, such as the centerof-mass location of the full configuration at $t_{f}$. However, we shall not investigate such possibilities here.

Again in analogy with the density-biased case described in Eqs. (4) -(6), we generate a Markov chain of histories $Y\left(t_{\mathrm{MC}}\right)$, with updates from $Y\left(t_{\mathrm{MC}}\right)$ to $Y\left(t_{\mathrm{MC}}+\right.$ $1)$ following the generation of trial configurations $Y_{\text {trial }}$, 


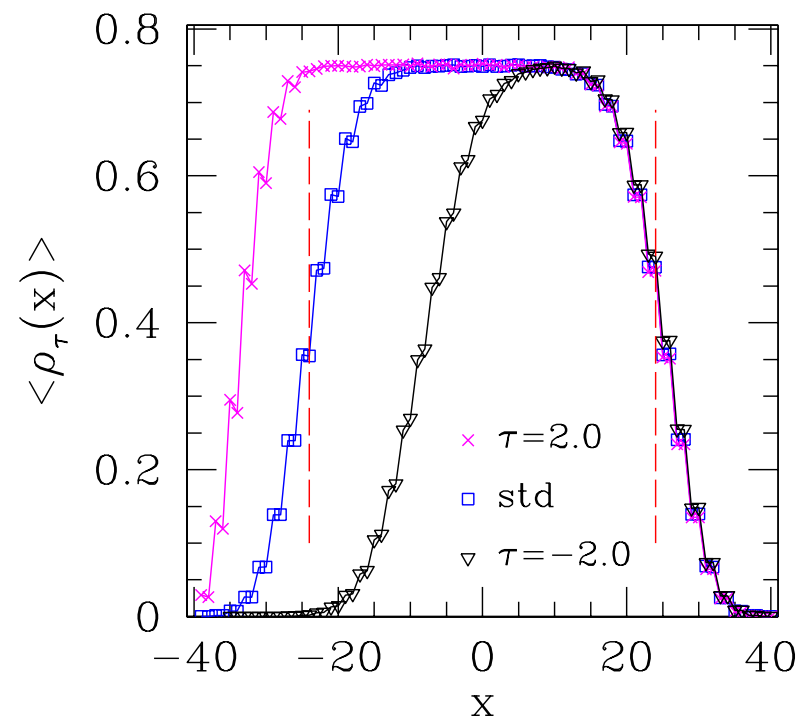

Figure 9. Ensemble-averaged local site occupations $\left\langle\rho_{\tau}(x)\right\rangle$ at $t_{f}=40$ against position $x$. The "std" label denotes unbiased sampling, see Fig. 8 Curves labeled $\tau=2.0$ and -2.0 correspond to biased sampling, see Eqs. (12), (13). $10^{7}$ independent samples in all cases. Dashed red lines mark boundaries of light cone at $x= \pm v_{B} t_{f}= \pm 24$.

for which the acceptance probability $A_{\tau}$ is

$$
A_{\tau}\left(Y \rightarrow Y_{\text {trial }}\right)=\min \left(1, e^{-\left\{x_{1 \mathrm{~m}}^{f}\left(Y_{\text {trial }}\right)-x_{1 \mathrm{~m}}^{f}\left[Y\left(t_{\mathrm{MC}}\right)\right]\right\} / \tau}\right) .
$$

Fig. 9 shows the results of sampling from biased ensembles, according to Eqs. (12) and (13), for both positive and negative values of $\tau$. For $\tau=2.0$ one gets $f_{\text {out }}^{L}=0.1489 \ldots$, to be compared to $f_{\text {out }}^{L}=0.03609 \ldots$ for the unbiased case. The biased-sampling scheme thus introduced indeed affects the overall density outside the light cone, as was the original intention. For biased cases, see the small breakdown of density degeneracy within pairs of neighboring sites, remarked upon earlier.

For a given $\tau$ the transformation between the biased distribution $P_{\tau}\left(x_{l m}^{f}\right)$ and the unbiased one goes along the same lines as that given in Eq. (5) for the case of $P_{\theta}\left(N_{f}\right), P\left(N_{f}\right)$. However, in order to make a similar transformation reconstructing the unbiased $\left\langle\rho_{\text {std }}(x)\right\rangle$ of Fig. 9 from $\left\langle\rho_{\tau}(x)\right\rangle$, further steps are needed.

At a given $x$ the ensemble-averaged density takes contributions from many biased histories $Y$, each of which has its own value of $x_{1 \mathrm{~m}}^{f}$ and, associated with it, an exponential weight factor which depends on $x_{1 \mathrm{~m}}^{f} / \tau$. There is also an overall normalization factor $Z_{\operatorname{lm}}(\tau)$, see Eq.(12). Thus one must keep track of the individual contributions, for all $x$, of each biased history $Y$ with its own $x_{1 \mathrm{~m}}^{f}$ and reweight them with the exponential factor $e^{x_{1 \mathrm{~m}}^{f} / \tau}$. The normalization factor can be numerically adjusted at the

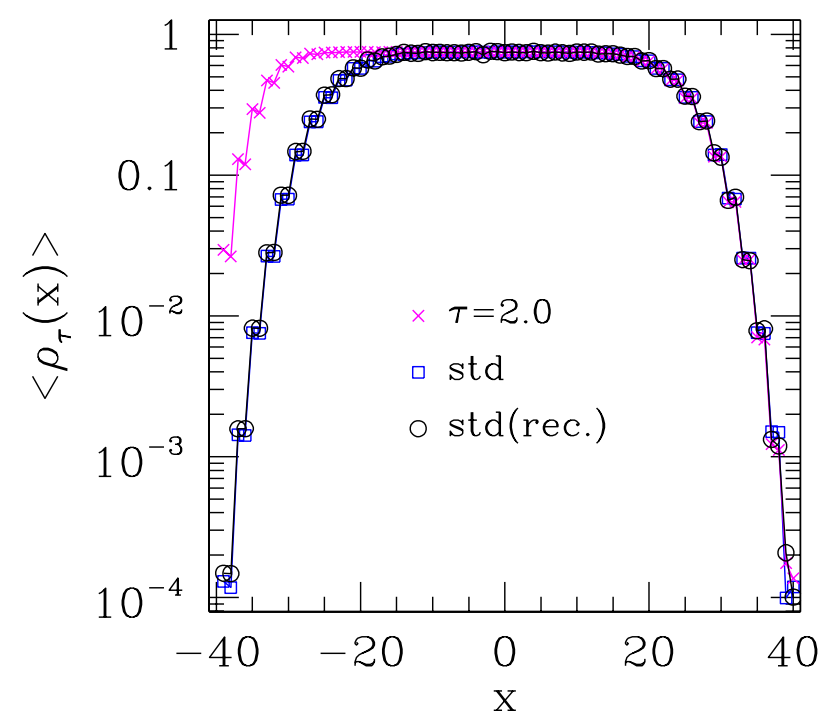

Figure 10. Ensemble-averaged local site occupations $\left\langle\rho_{\tau}(x)\right\rangle$ at $t_{f}=40$ against position $x$. The "std" label denotes unbiased sampling, while the curve labelled $\tau=2.0$ corresponds to biased sampling, see Fig. 9 "std(rec.)" denotes $\left\langle\rho_{\text {std }}(x)\right\rangle$ reconstructed from $\left\langle\rho_{\tau}(x)\right\rangle, \tau=2.0$ (see text). Note logarithmic scale for the vertical axis.

end.

We proceeded as just described, for the samples used in Fig. 9 for $\tau=2$, and reconstructed $\left\langle\rho_{\text {std }}(x)\right\rangle$, see the curve denoted by "std(rec.)" in Fig. 10

One sees in Fig. 10 that even close to the cutoff at $x=-40$, the averaged densities predicted either by the unbiased curve or by the reconstructed one coincide to within $1-2 \%$ : both are of order $10^{-4}$. So, $\left\langle\rho_{\text {std }}(x)\right\rangle$ is already rather accurate for $|x| \sim t_{f}$.

The reasons for this are: (i) there is a hard limit to the range of $x$ beyond which particles are not allowed at all, namely $x= \pm t_{f}$; and (ii) the average local density everywhere results from collecting samples without restrictions over all accepted histories $Y$, each of which may contribute to occupation near the cutoff regardless of its $N_{f}$. Compare this, e.g., to $P\left(N_{f}\right)$ for small $N_{f} \sim 1$. Owing to the update rules, final configurations with very few particles are extremely infrequent, so the overwhelming majority of histories do not contribute to that in unbiased sampling.

\section{Configurations with $N_{f}=1$ : bottlenecks}

We now turn to the discussion of morphological features of the connected set of successive configurations assumed by the cluster of occupied sites, as it evolves from $t=0$ to $t_{f}$ according to the $1+1-\mathrm{D}$ RUC growth rules given in Eq. (2). In what follows, such set will be referred 
to as a configuration, for short. Concerning the subset of processes which end with a single particle at the final time $t_{f}$, for the Ising case $q=2$ of interest here it has been predicted [13] that a phase transition takes place in the shape of the associated configurations: from "fat", i.e. a compact shape, for configurations ending within the light cone $\left(x_{f}<v_{B} t_{f}\right)$, to "thin", i.e. exhibiting a large density of string-like portions or "bottlenecks", for super-luminar ones $\left(x_{f}>v_{B} t_{f}\right)$.

In what follows we adapt the ideas developed in Secs. IIIC 1 and IIIC 2 to the numerical investigation of such predictions.

One needs to enhance the probability of obtaining the right sort of configuration, namely one (i) with a single particle left at the end, and (ii) preferably outside the light cone. It has been shown respectively in Secs. IIIB and III 2 2 how either requisite can be fulfilled separately.

We propose the simplest possible ad hoc generalization, namely assuming a multiplicative form for the Boltzmann-like factor in the Metropolis update scheme. That is,

$$
P\left(N_{f}, x_{\operatorname{lm}}^{f}\right) \propto \exp \left[-\left(N_{f} / \theta\right)-\left(x_{\operatorname{lm}}^{f} / \tau\right)\right],
$$

see Eqs. (41) and (12).

To show that Eq. (14) is appropriate for our purposes in this Section, first note that we will be confining ourselves to the restricted ensemble of configurations with $N_{f}=1$; of course the location of that particle will be $x_{f}=x_{\mathrm{lm}}^{f}$. As already argued in connection with Figure 7 the factor $\exp (-1 / \theta)$ gives the same bias to all final configurations being considered (and different from that for the ones with $N_{f}>1$ ). Within this subset, those with the single particle further to the left are more favored; those configurations sharing the same $x_{\mathrm{lm}}^{f}$ are equally biased by the $\tau$ - dependent factor.

This is convenient, as the relative probability of occurrence of any two configurations in the sub-sub-set with (i) $N_{f}=1$ and (ii) sharing the same $x_{\mathrm{lm}}^{f}$ is then, similarly to the case of Figure 7 , the same as the ratio of their natural (unbiased) probabilities of occurring.

Having this in mind, we ran simulations with the Metropolis probability according to Eq. (14) above. We used two sets of $(\theta, \tau)$, namely $(0.6,1.5)$ and $(0.4,1.0)$. So, particles are pulled to the left of $x=0$ (where the initial particle is).

Fig. 11] shows two selected configurations with $t_{f}=40$ and $N_{f}=1$, one sub-luminar and the other superluminar. It can be seen that in both cases the qualitative features predicted in Ref. [13] are unequivocally present: the super-luminar (blue) configuration consists mostly of string-like segments, while the sub-luminar (magenta) one exhibits a broad hull almost everywhere except close to $t=0$ and $t_{f}$ (though with a few internal voids).

We studied the statistics of bottlenecks, i.e., the number $N_{\text {bn }}$ of constant- $t$ cross-sections of a given $N_{f}=1$ configuration (other than the $t=0$ and $t=t_{f}$ ones) which have a single particle.

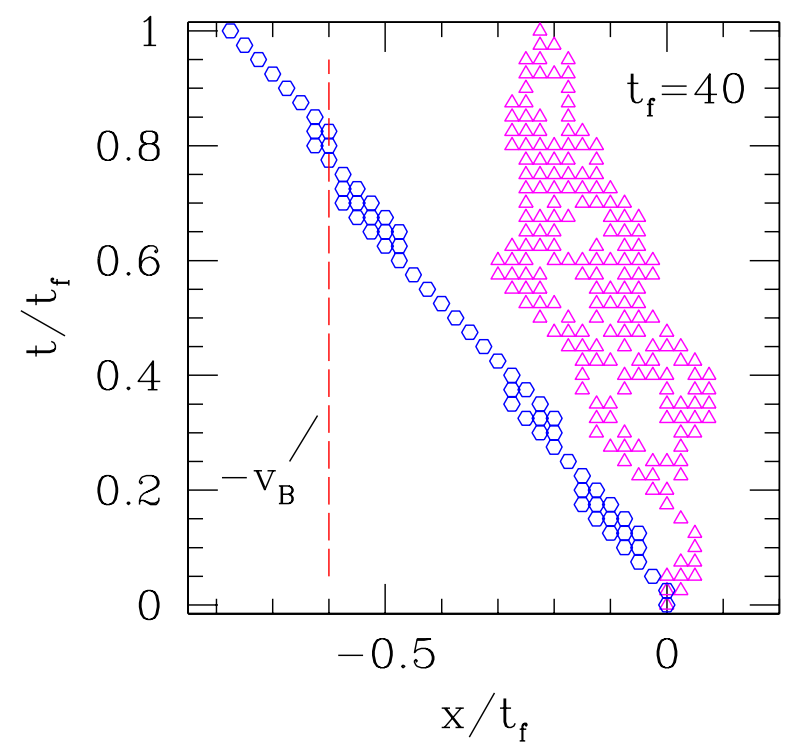

Figure 11. Spacetime diagrams representing two configurations with $N_{f}=1$, one sub-luminar (magenta triangles) and the other super-luminar (blue hexagons), taken from a simulation with $\theta=0.6, \tau=1.5$, see Eq. (14), and $t_{f}=40$.

In Figure 12 the vertical axis gives the ensembleaveraged number $\left\langle N_{\mathrm{bn}}\right\rangle$ of bottlenecks according to (scaled) final position of single particle $x_{f} / t_{f}$, for $t_{f}=40$, 60, 80, and 100 .

For each value of $t_{f}$ we generated $10^{9}$ samples in total, for each of the $(\theta, \tau)$ pairs mentioned above. For all sets of $\left(t_{f}, \theta, \tau\right)$ approximately $10^{7}$ turned out to have $N_{f}=1$. Because of the low value of the directional-bias temperature, essentially all $N_{f}=1$ configurations have $x_{f}<0$ in this case. Even so, we have found that those lying outside the light cone are in a clear minority. For the data depicted in Figure 12 the largest fraction of $N_{f}=1$ configurations which are super-luminar is $\approx 0.7 \%$, for $t_{f}=40$. Note that, due to the normalization used, for a given $t_{f}$ it is appropriate to mix $\left\langle N_{\mathrm{bn}}\right\rangle$ data for both pairs of biasing parameters. We have seen that results for $(\theta, \tau)=(0.4,1.0)$ behave smoothly for intervals of $x_{f} / t_{f}$ generally farther from the origin than those corresponding to $(0.6,1.5)$, though with a reasonably broad intermediate range where such intervals intersect (the respective data coinciding with one another within small fluctuations).

Figure 12 shows that $\left\langle N_{\mathrm{bn}}\right\rangle$ increases when $\left|x_{f}\right|$ approaches $v_{B} t_{f}$, and (for finite $t_{f}$ ) shows a trend to keep increasing beyond that; larger values of $t_{f}$ are associated with steeper growth rates for $\left\langle N_{\mathrm{bn}}\right\rangle$. This is in qualitative agreement with the existing prediction of a fatto-thin transition at $x_{f}=v_{B} t_{f}$ [13]. Defining $\left\langle N_{\mathrm{bn}}\right\rangle_{c}$ as the value of $\left\langle N_{\mathrm{bn}}\right\rangle$ at the (assumedly critical) position $x_{f} / t_{f}=-v_{B}$, the inset of Fig. 12 depicts $\left\langle N_{\mathrm{bn}}\right\rangle_{c}^{-1}$ against $t_{f}^{-1}$. The dashed line is a parabolic fit of the 


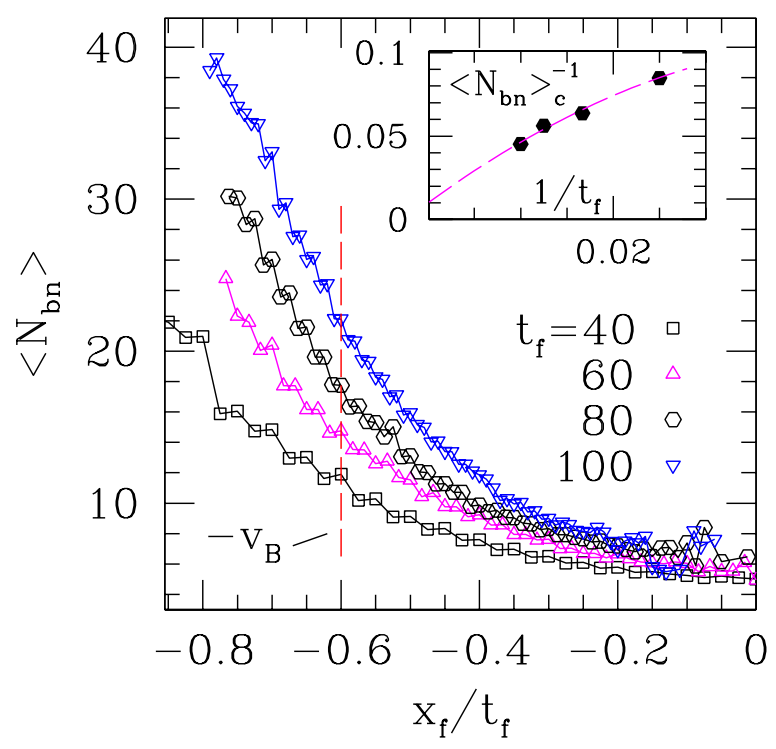

Figure 12. Main Figure: the vertical axis gives the average number $\left\langle N_{\mathrm{bn}}\right\rangle$ of bottlenecks according to (scaled) final position of single particle $x_{f} / t_{f}$, for $t_{f}=40,60,80$, and 100. Averages are taken over the restricted ensemble of configurations with $N_{f}=1$. Data were generated with $(\theta, \tau)=(0.6,1.5)$ and $(0.4,1.0)$, see Eq. (14) and text. Inset: points are $\left\langle N_{\mathrm{bn}}\right\rangle_{c}^{-1}$ [ (inverse) $\left\langle N_{\mathrm{bn}}\right\rangle$ at $x_{f} / t_{f}=-v_{B}$ ]; line is parabolic fit of data against $1 / t_{f}$ (see text).

data. Of course, adjusting four points with three free parameters is only an attempt to probe for underlying trends, the results of which should be considered with due caution. Estimating an uncertainty of order $4 \%$ for each data point, the extrapolated intercept of the vertical axis is $\lim _{t_{f}^{-1} \rightarrow 0}\left\langle N_{\mathrm{bn}}\right\rangle_{c}^{-1}=0.01 \pm 0.02$, with $\chi_{\text {dof }}^{2}=1.8$. Thus we find indications which are broadly compatible with the existence of a second-order transition for $\left\langle N_{\mathrm{bn}}\right\rangle$ at $x_{f} / t_{f}=-v_{B}$, for configurations with $N_{f}=1$.

In summary, our results indicate the applicability of studying the statistics of bottlenecks to provide numerical evidence concerning the predicted fat-to-thin transition.

\section{DISCUSSION AND CONCLUSIONS}

We have studied the statistics of selected rare events in RUC evolution processes. To this end, we have applied a large-deviation approach whose general characteristics are well-established [8-11]. In order to take into account some specific features of RUC evolution, two main adaptations are needed.

Firstly, the procedure of replacing a single RN of the vector list $\xi$, defined in Sec. III may have effects throughout the extension of the light cone associated with the bond where it occurs. Thus the "local spin-flip" analogy invoked in previous applications is not obviously applicable. Even so, we verified that rules (i)-(iii) given at the end of Sec. II work well here. The main difference to other cases in the literature is that, in order to obey the rule of thumb of having approximately $50 \%$ of the trial histories accepted [11], one needs change only between $1.25 \%$ and $2.5 \%$ of the components of $\xi$, to be compared to the usual $10 \%$ or so used elsewhere [8-11].

Also, in our simulations of RUC evolution the process always starts with the same configuration, namely $n_{\ell}(t=$ $0)=\delta_{\ell, 0}$. So, with standard (unbiased) sampling there is no need to wait for "thermalization". On the other hand, for biased sampling a distinct sort of equilibration takes place. Fig. 3 illustrates that for finite temperature bias $\theta$, it takes a finite amount of Monte Carlo time $t_{\mathrm{MC}}$ for the path followed by the successive $Y\left(t_{\mathrm{MC}}\right)$ to lead the system to the appropriate region of configuration space.

By incorporating the adaptations just recalled, we produced accurate estimates of $P_{1}\left(t_{f}\right)$ as low as $10^{-40}$ for $t_{f}=200$, which is a suitably large value of $t_{f}$ for our purposes here. Remarkably, we have found the actual distribution $P_{1}\left(t_{f}\right)$ to differ only slightly from a Gaussian shape. Fig. 6] shows that using the latter approximation results in a mismatch of at most one order of magnitude out of 40 (for $P_{1}\left(t_{f}\right)$ at $t_{f}=200$ ).

Regarding super-luminar configurations, Figs. 7 and 8 illustrate that these are in general very unlikely to occur. In order to increase the number of realizations with occupied sites outside the light cone, we introduced in Eq. (12) a new temperature-like bias parameter $\tau$ associated with a directional bias.

For some quantities of interest, the form Eq. (12) is especially convenient when used together with the idea of analyzing restricted ensembles in which the bias factor is the same for all samples considered. Thus their relative probability is unchanged (this latter concept was first used here in connection with the unbiased-sampling data of Fig. (7). One then has the latitude to employ ad hoc schemes via Eqs. (12) and (13), to evaluate suitable averages with no need to work back to the original distribution.

It can be seen, for example, that the ensemble-averaged density distributions shown in Fig. 9 tend to coincide at the right end because the Boltzmann-like factor in Eq. (12) was defined as depending only on the position of the leftmost particle at $t_{f}$. Considered on its own, such feature could be deemed as a mere artifact. However, the same definition comes to one's advantage in Sec. III C3. where one is looking at the subset of configurations (i) with a single particle at the end and (ii) outside and, say, on the left of, the light cone. In this particular case, as explained in Sec. III C3 the choice made ensures that the configurations used in evaluating bottleneck numbers for Fig. 12 have the correct relative weights. See Eq. (14).

Finally, Figs. 11 and 12 lend numerical support to the prediction of a fat-to-thin transition for the subset of configurations ending with a single particle [13], depending on where the last particle ends up relative to the light 
cone.

\section{ACKNOWLEDGMENTS}

The author thanks the Rudolf Peierls Centre for Theoretical Physics, Oxford, for hospitality during his visit, and Adam Nahum for many enlightening discussions and suggestions. This study was financed in part by Coordenação de Aperfeiçoamento de Pessoal de Nível Superior - Brasil (CAPES) - Finance Code 001.
[1] A. I. Larkin and Yu. N. Ovchinnikov, Sov. Phys. JETP 28, 1200 (1969).

[2] A. Nahum, S. Vijay, and J. Haah, Phys. Rev. X 8, 021014 (2018).

[3] F. den Hollander, Large Deviations (American Mathematical Society, Providence, RI, 2000).

[4] H. Touchette, Physics Reports 478, 1 (2009).

[5] A. Dembo and O. Zeitouni, Large Deviations Techniques and Applications (Springer, Berlin, 2010).

[6] J. M. Hammersley and K. W. Morton, Math. Proc. Cambr. Phil. Soc. 52, 449 (1956).
[7] J. A. Bucklew, Introduction to Rare Event Simulation (Springer-Verlag, New York, 2004).

[8] A. K. Hartmann, Phys. Rev. E 65, 056102 (2002).

[9] G. E. Crooks and D. Chandler, Phys. Rev. E 64, 026109 (2001).

[10] A. K. Hartmann, Phys. Rev. E 89, 052103 (2014).

[11] W. Staffeldt and A. K. Hartmann, Phys. Rev. E 100, 062301 (2019).

[12] A. K. Hartmann, Eur. Phys. J. B 84, 627 (2011).

[13] A. Nahum et al, unpublished (2020). 